\title{
Applications of laser printing for organic electronics
}

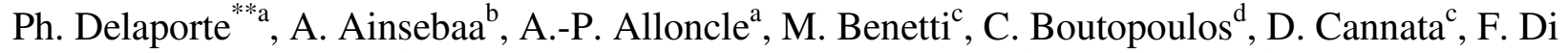 \\ Pietrantonio $^{c}$, V. Dinca ${ }^{\mathrm{e}, \mathrm{f}, \mathrm{g}}$, M. Dinescu ${ }^{\mathrm{e}}$, J. Dutroncy ${ }^{\mathrm{h}}$, R. Eason ${ }^{\mathrm{i}}$, M. Feinaugle ${ }^{\mathrm{i}}$, J.-M. $^{-}$. Fernández-

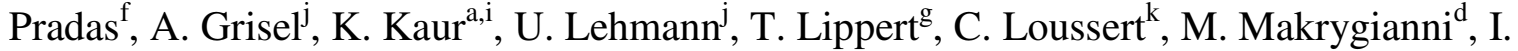 \\ Manfredonia, T. Mattle ${ }^{\mathrm{g}}$, J.-L. Morenza ${ }^{\mathrm{f}}$, M. Nagel ${ }^{\mathrm{m}}$, F. Nüesch ${ }^{\mathrm{m}}$, A. Palla-Papavlu ${ }^{\mathrm{e}, \mathrm{f}, \mathrm{g}}$, L. Rapp ${ }^{\mathrm{a}}$, N. \\ Rizvi $^{\mathrm{n}}$, G. Rodio ${ }^{1}$, S. Sanaur ${ }^{\mathrm{b}}$, P. Serra ${ }^{\mathrm{f}}$, J. Shaw-Stewart ${ }^{\mathrm{m}}$, C. L Sones ${ }^{\mathrm{i}}$, E. Verona ${ }^{\mathrm{c}}$, I. Zergioti ${ }^{\mathrm{d}}$ \\ ${ }^{a}$ Aix-Marseille University, CNRS LP3 laboratory, France; ${ }^{b}$ Ecole Nationale Superieure des Mines, \\ Dept Bioelect, Ctr Microelect Provence, Gardanne, France; 'Istituto di Acustica "O.M. Corbino", \\ CNR, Roma, Italy; ${ }^{\mathrm{d}}$ Physics Department, National Technical University of Athens, Athens, Greece; \\ ${ }^{\mathrm{e}}$ National Institute for Lasers, Plasma and Radiation Physics, Magurele, Bucharest, Romania; \\ ${ }^{\mathrm{f}}$ Department of Applied Physics and Optics, University of Barcelona, Barcelona, Spain; ${ }^{\mathrm{g}}$ Paul \\ Scherrer Institut, General Energies, Villigen-PSI, Switzerland; ${ }^{\mathrm{h}}$ ACXYS Technology, St Martin le \\ Vinoux, France; ${ }^{\mathrm{i} O p t o e l e c t r o n i c s ~ R e s e a r c h ~ C e n t r e, ~ U n i v e r s i t y ~ o f ~ S o u t h a m p t o n, ~ S o u t h a m p t o n, ~}$ \\ United Kingdom; ${ }^{\mathrm{j}}$ MICROSENS, Neuchatel, Switzerland; ${ }^{\mathrm{K}}$ TAGSYS RFID, La penne / Huveaune, \\ France; ${ }^{1}$ BIOSENSOR srl, Formello, Rome, Italy; ${ }^{\mathrm{m}}$ EMPA, Functional Polymers, Düebendorf, \\ Switzerland; ${ }^{\mathrm{L}} \mathrm{LML}$, Denbigshire, United Kingdom.
}

\begin{abstract}
The development of organic electronic requires a non contact digital printing process. The European funded e-LIFT project investigated the possibility of using the Laser Induced Forward Transfer (LIFT) technique to address this field of applications. This process has been optimized for the deposition of functional organic and inorganic materials in liquid and solid phase, and a set of polymer dynamic release layer (DRL) has been developed to allow a safe transfer of a large range of thin films. Then, some specific applications related to the development of heterogeneous integration in organic electronics have been addressed. We demonstrated the ability of LIFT process to print thin film of organic semiconductor and to realize Organic Thin Film Transistors (OTFT) with mobilities as high as $410^{-2} \mathrm{~cm}^{2} . \mathrm{V}^{-1} \cdot \mathrm{s}^{-1}$ and $\mathrm{I}_{\mathrm{on}} / \mathrm{I}_{\text {off }}$ ratio of $2.810^{5}$. Polymer Light Emitting Diodes (PLED) have been laser printed by transferring in a single step process a stack of thin films, leading to the fabrication of red, blue green PLEDs with luminance ranging from $145 \mathrm{~cd} . \mathrm{m}^{2}$ to $540 \mathrm{~cd} . \mathrm{m}^{-2}$. Then, chemical sensors and biosensors have been fabricated by printing polymers and proteins on Surface Acoustic Wave (SAW) devices. The ability of LIFT to transfer several sensing elements on a same device with high resolution allows improving the selectivity of these sensors and biosensors. Gas sensors based on the deposition of semiconducting oxide $\left(\mathrm{SnO}_{2}\right)$ and biosensors for the detection of herbicides relying on the printing of proteins have also been realized and their performances overcome those of commercial devices. At last, we successfully laser-printed thermoelectric materials and realized microgenerators for energy harvesting applications.
\end{abstract}

Keywords: LIFT, laser printing, organic electronics, OTFTs, PLEDs, sensors, biosensors, energy harvesting

\section{INTRODUCTION}

For many applications within the microelectronic industry, the challenge is no longer one of pitch size reduction. The most relevant keywords are now 'flexible', 'low cost' and 'large area'. The typical dimension of the elementary unit is of the order of a few microns ( 3 to $200 \mu \mathrm{m}$ ). Moreover, the integration or many functions on the same device via one unique process is of paramount importance. Organic compounds currently present very interesting performance and are

* delaporte@lp3.univ-mrs.fr; phone +33-491 829 284; fax +33-491 829 289; www.lp3.univ-mrs.fr 
becoming increasingly suitable for device production. Two approaches are developed to manufacture these printed electronic devices. First, mass printing technologies (gravure printing, flexography, screen printing ...) which are well known from the graphic arts industry and enable roll to roll or high speed sheet to sheet processing. Second, the noncontact and digital printing methods, especially ink-jet printing which has become widespread in the graphics industry, are seeing significant use for organic and printed electronics. The first limitation of roll-to-roll processes is the lack of flexibility. The impression cylinder is designed for only one application, and any modification in the design requires the production of a new cylinder. The inkjet printing is some more flexible but the process is limited by the lateral resolution ( around $50 \mu \mathrm{m}$ ) and by the dispensing speed due to mechanical constraints such as drop viscosity, and mechanical displacement of the printing heads. All these conventional techniques require the use of liquid inks and the quality of the deposition is critically dependant of the ink properties, notably viscosity, adhesion and separation properties and drying which all have a strong influence on the stability, shape and lateral resolution of the deposit.

In this context, the development of a simple process allowing the deposition of a wide variety of materials, with high spatial resolution is of great interest for the manufacturing of future smart organic electronic devices. The objective of the e-LIFT European project (FP7 ICT-247868) is to demonstrate the integrability of a new laser process in the industrial world of electronic device manufacturing for the localized deposition of organic and inorganic materials under liquid or solid format. Laser direct-write technologies, such Laser-Induced Forward Transfer (LIFT) ${ }^{1,2}$ appear very promising these applications.

The LIFT technique had been successfully used for the functional deposition of deoxyribonucleic acid (DNA) ${ }^{3}$, active optical structure ${ }^{4,5}$, polymer $^{6}$, biomaterials ${ }^{7,8}$, nanotubes ${ }^{9}$, metals ${ }^{10}$, nanoparticles ${ }^{11-13}$ and electronic devices such as organic transistors (OTFT) ${ }^{14}$ and organic light emitting diode (OLED) $)^{15}$. This process is a promising alternative for fabrication of organic and metallic electronic components. This single step, direct printing technique offers the ability to make surface micro patterning or localized deposition of material. It can be applied to sensitive materials without altering their properties but it also allows to direct-write multilayer systems in a solvent-free single step, without requiring any shadowing mask or high vacuum installation.

First, we performed the optimization of the LIFT process for representative materials and substrates (flexible and rigid) in order to solve the potential technological blocking points and to determine the process windows. Then, in order to validate the process, some specific applications have been addressed and that led to the realization and characterisation of devices like OTFTs, OLEDs, sensors, biosensors and energy harvesters.

\section{KEY FEATURES OF LIFT PROCESS}

Since the first experiment of LIFT performed by Bohandy et al. ${ }^{1}$ for the deposition of copper film on fused silica substrate in vacuum using an ArF excimer laser $(193 \mathrm{~nm})$, many studies have been done on this process. Several presentations of this technique $e^{2,16,17}$ as well as its potentiality for printing different materials ${ }^{18,19}$ and devices ${ }^{20}$ are available in literature and the following description of the LIFT principle is limited to the basic characteristics of the process. For printing pixels in solid phase, this technique consists of the irradiation, using a pulsed laser, of a thin layer of an absorbing material (the donor) that has been deposited onto a transparent substrate. The layer is irradiated through the substrate and the light-matter interaction which takes place at the interface generates a strong increase of the local pressure. As a result, a small pixel of the thin film is ejected from the substrate surface and deposited onto a target substrate (the receiver) arranged to be in close proximity to the donor substrate. The transfer of transparent layers is also possible with this technique by placing a thin layer of absorbing material called a dynamic release layer (DRL) between the substrate and the film required for deposition. The size of the ejected material is controlled by the size of the incident laser spot. LIFT enables the deposition of a large range of materials with typical sizes of a few micrometers. For printing droplets in liquid phase, the donor film consists in a liquid layer with a thickness of few micrometers. The laser interacts with a DRL or the liquid layer if it is absorbing enough, and that generates the formation of a vapor bubble which expands towards the surface of the liquid. This process drags the liquid around the bubble to form a very thin and stable jet which reaches the surface and form a droplet.

\section{1 printing in solid phase}

The influence of different laser irradiation parameters on the physics of LIFT has been investigated. The only interest of varying the wavelength is to obtain a high absorptivity of the material to be transferred. When the polymer DRL developed in the frame of this project is used, laser wavelength below $220 \mathrm{~nm}$ or in the range $280-350 \mathrm{~nm}$ is required. No significant influence of the pulse duration (from 100fs to 30ns) on the printing process has been observed. The 
hydrodynamics of the pixel ejection can be slightly modified when varying the pulse duration ${ }^{21,22}$, but we never found a material for which the pulse duration is a limiting parameter to achieve a safe printing. The laser fluence is a very important process and need to be tuned for each material and thickness. Too low fluence does not allow a complete transfer of the layer, and too high fluence leads to damaged pixels or debris generation. As a consequence, the deposition of a uniform pixel requires a uniform energy profile of the laser beam, typically with fluctuations lower than $10 \%$.

Two more critical parameters, also closely linked to each other, are the surrounding gas pressure and the distance between the receiver and the donor substrates. As previously observed ${ }^{23,24,25}$, the irradiation of thin donor film leads to the formation of a shock wave flying before the ejected pixel. However, as we can see on the figure 1, when this shock wave reaches the receiver substrate, it reflects back and interacts with the pixel. This interaction stops the pixel motion and often destroys $\mathrm{it}^{26}$. Then, laser printing at atmospheric pressure can only be successful when working with no gap between receiver and donor substrates, or with a gap smaller than few micrometers and an accurate selection of the fluence. To avoid the generation of this shock wave, printing experiments have been performed under vacuum, but the velocity of the flyer was so high that it breaks when reaching the receiver substrate. Some studies have been performed at reduced pressure to determine the relation between the donor - receiver distances allowing a safe printing and the the pressure of the surrounding gas. Figure 2 summarizes these results. It is clear that working under pressure lower than few tens of mbar allows printing pixels with a wide range of fluences and distance between receiver and donor as large as $40 \mu \mathrm{m}^{27}$. The constraint of printing at reduced pressures of tens of mbar is not too strong for industrial applications and it gives more flexibility on the donor-receiver gap.

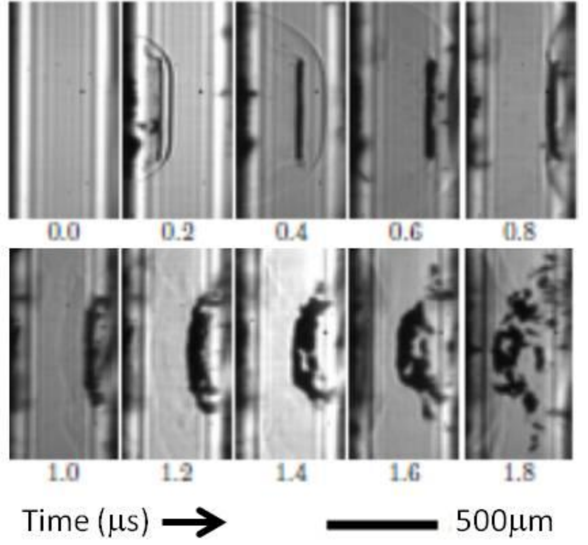

Figure 1: Sequence of pictures taken with a $350 \mathrm{~nm}$ TP / $80 \mathrm{~nm} \mathrm{Al}$ sample at $160 \mathrm{~mJ} / \mathrm{cm}^{2}$ with a receiver substrate at a distance of $0.5 \mathrm{~mm}$.

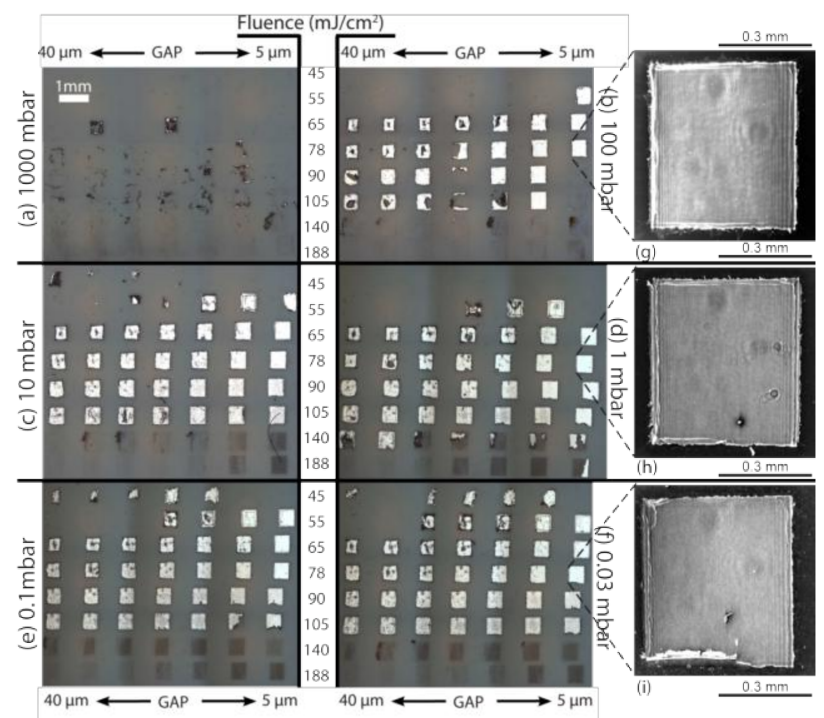

Figure 2: Aluminum pixel arrays printed at different pressures, from $1000 \mathrm{mbar}$ (a) to $0.03 \mathrm{mbar}$ (f). The columns correspond to gaps varying from 5 to $40 \mu \mathrm{m}$. The lines correspond to fluence varying from 45 to $188 \mathrm{~mJ} / \mathrm{cm}^{2}$. The donor film was made of 350 $\mathrm{nm}$ TP / $80 \mathrm{~nm} \mathrm{Ag} \mathrm{/} 80 \mathrm{~nm}$ PFO.

\section{2 printing in liquid phase}

A clear view of the mechanisms of the LIFT of liquid is required to understand the influence of the different parameters on the printing process. Time-resolved fast imaging is the best approach to study this process and many studies have been performed on this topics ${ }^{28-31}$. To summarize the mechanisms, the laser interacts with a titanium DRL or the liquid layer if it is absorbing enough, and that generates the formation of a vapor bubble which expands towards the surface of the liquid. This process drags the liquid around the bubble to form a very thin and stable jet which can reach the surface and form a droplet. These hydrodynamics mechanisms are strongly dependant of the viscosity and the thickness of the liquid film as well as the energy and the spot size of the laser.

Two kinds of liquid have been used to investigate the physics of LIFT of liquids: water based solutions to simulate the biomaterials that have to be printed for the realization of biosensors and silver nanoparticle inks for the optimization of the electronic applications. For the biological materials printing, water and glycerol was mixed to vary the viscosity as 
well as sodium dodecil sulfate (SDS) as surfactant agent. The specifications of the inks were the following: $20 \%$ wt of silver content, viscosity of $10-30 \mathrm{mPa} . \mathrm{s}$, density of $1.25 \mathrm{~g} / \mathrm{cm}^{3}$, nanoparticle size in the range of $80-110 \mathrm{~nm}$, alcohol based solvent. From these studies we can conclude that there is no restriction concerning the choice of the wavelength (193$1027 \mathrm{~nm}$ ) and pulse duration ( $450 \mathrm{fs}-10 \mathrm{~ns}$ ), and that the gap between donor and receiver substrates does not constitute any significant constraint for the process from $20 \mu \mathrm{m}$ to $2 \mathrm{~mm}$. Well-defined droplets with sizes between $20 \mu \mathrm{m}$ and several hundreds of microns have been printed for liquid viscosities varying from $1 \mathrm{mPa} . \mathrm{s}$ to about $1000 \mathrm{mPa} \cdot \mathrm{s}^{12,29}$.

Experimental studies have also been performed to optimize the printing of lines from liquid. Figure 3 shows the morphologies of lines laser-printed with different overlap values. Droplets start merging as soon as the centre to centre distance is equal to the droplet diameter, and we observe scalloping effect on the printed lines until the overlap reaches $50 \%$. For this value, we observed a very straight and uniform line. Further increase of the overlap leads to the formation of wider zones along the line. That is interesting to notice that, whatever the conditions we have investigated, it has been possible to print uniform line, and the optimum conditions of overlap were always close to $50 \%$.

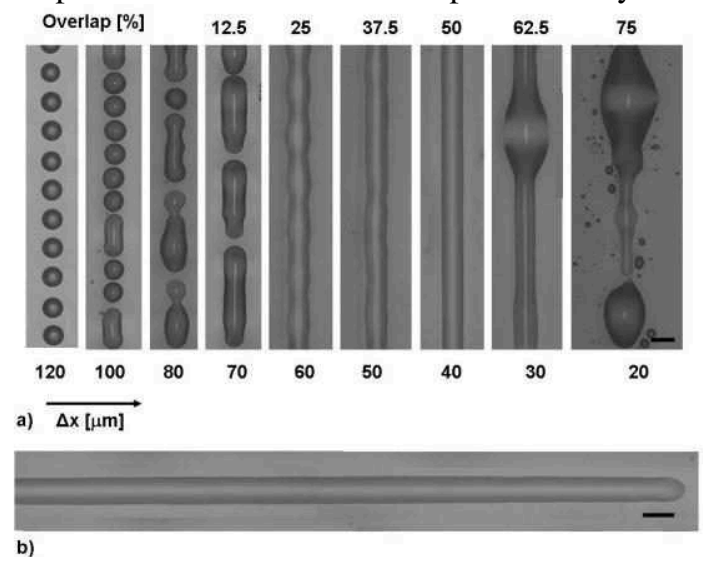

Figure 3: Optical microscope images of laser-printed lines of water-glycerol mixture $(50 \% \mathrm{v} / \mathrm{v}-6.5 \mathrm{mPa} . \mathrm{s})$. (a) Influence of overlap distance on line morphology. (b) $2 \mathrm{~mm}$ long line.

\subsection{Optimization of polymer DRL}

The transfer of material with laser process requires the absorption of laser energy and its transformation into mechanical energy. In case of transparent film, we developed a triazene polymer layer ${ }^{32-34}$, namely dynamic release layer (DRL), which is deposited between the transparent substrate and the film to be printed. Another important effect of this layer is to protect the film against any photochemical or thermal damage that could be induced by a direct irradiation. Different optimization steps have been developed regarding: the tuning of the absorption coefficient of the DRL versus the laser wavelength, the vaporization of the layer under irradiation to avoid the generation of debris, the development of DRLs with different solvents to make possible its use for all the materials ${ }^{35}$.

The basic structure of such photopolymers is shown in figure 4. The photocleavable aryltriazene chromophore is covalently incorporated into the polymer main chain. The thermodynamic driving force of the photo-triggered fragmentation mechanism is the formation of elemental nitrogen upon homolytical cleavage of the triazene moiety. The type of the bridge $X$ has an influence on the absorption range, whereas the length of the spacer unit $Y$ determines the number of chromophores along the backbone, and therefore the absorption coefficient. The side chains $R$ at the triazene unit have an influence on the packing behavior of the polymer chains and allow the introduction of further functional groups in order to tailor the application properties as e.g. solubility in different solvents and the polarity.
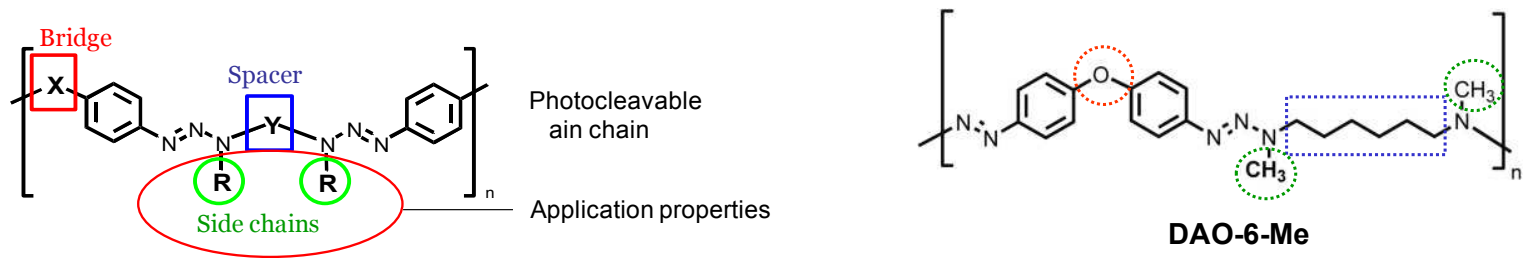

Figure 4: General chemical structure of aryltriazene photopolymers and as example the derivative DAO-6-Me that is frequently used as a reference material for LIFT studies. Here, the bridge $\mathrm{X}$ is an ether $(\mathrm{O})$, the spacer $\mathrm{Y} \mathrm{a}_{6}$-alkyl chain and the side-chains $\mathrm{R}$ small methyl groups. 


\subsection{Beam shaping}

The studies performed in the frame of the project demonstrated that the printing in solid phase requires a uniform energy beam profile. However, the ejection of the pixel from the donor substrate is based on a mechanical effect which breaks the film. So the ability of laser printing to deposit pixels with straight edges depends on beam profile, but also on material properties (hardness, fracture toughness, tensile strength, ductility ...). The use of top hat beam profile can leads to the printing of pixels with edge variations, depending of the material characteristics. To overcome this drawback, we designed a smart beam shaping with higher energy at the edges of the pixel than in the centre. We shaped the beam of an ArF excimer laser to obtain a profile with over-intensities on the edges of the beam. The laser beam is first split in two parts. One part is used to shape the edges (a) and the other to shape the central part (b). Finally, the two arms are merged to form the final smart beam profile (c). The intensity ratio between the edges and the centre can be adjusted by means of a dual attenuation system. The energy in the edge of the beam is used to cut the film while the central part of the beam propels the pixel towards the receiver.

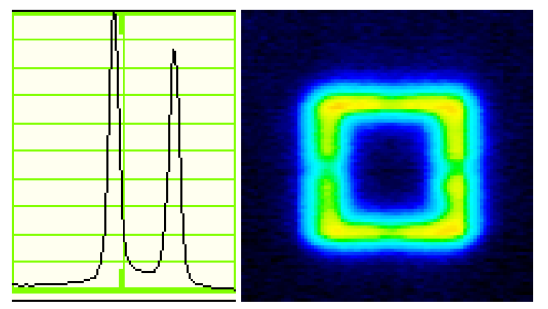

(a)

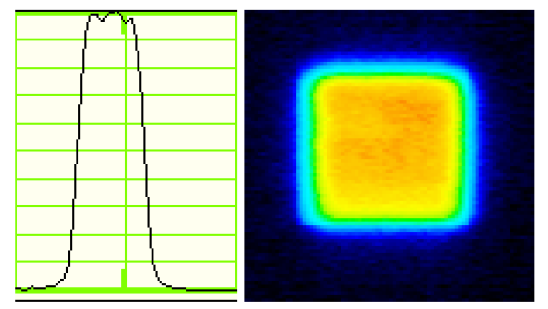

(b)

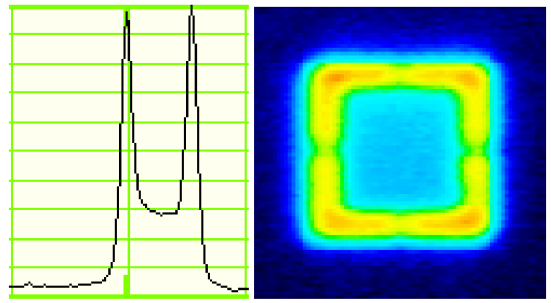

(c)

Figure 5: Energy profiles of shaped ArF laser beam: (a) beam 1 - edges, (b) beam 2 - centre, (c) beam $1+$ beam 2.

Pixels of organic material have been deposited with this smart beam shaping. The overall energy required to print a welldefined and undamaged pixel is lower when using the smart beam shaping compared to the top hat profile. The optimized energy repartition on the donor substrate allows the reduction by a factor of two of the laser fluence in the central part of the beam. One of the consequences is the reduction of the intensity of the shock wave generated by the pixel motion, which has a deleterious effect for the printing of pixel on the receiver substrate. This reduction of the laser fluence also limits the risk of material damage by the laser irradiation.

With the same purpose, we also used a focused ion beam to pre-machine the pixels before the laser transfer. This approach has been especially successful for thick $(1-3 \mu \mathrm{m})$ and brittle films such as crystal ${ }^{36}$ or piezoelectric materials ${ }^{37}$.

\section{APPLICATION TO POLYMER LIGHT EMITTING DIODES}

LIFT process has been used to print highly resolved RGB (red, blue green) pixels of polymer light emitting diodes (PLEDs) with high luminescence efficiency. Mainly Polyfluorene (PFO)-based polymers were studied as the electroluminescent material, according to the proposed concept to generate the green and red emission by blending triplet emitters as dopants into the PFO matrix ${ }^{38,39}$. Devices were based on the architecture glass / $140 \mathrm{~nm}$ ITO / $60 \mathrm{~nm}$ PEDOT:PSS / $40 \mathrm{~nm}$ PVK (Poly(9-vinylcarbazole) / $50 \mathrm{~nm}$ light-emitting polymer (LEP) / Cathode. The figure 6 shows the structure of both the receiver and donor substrates. For all of these experiments, a $\sim 15 \mu \mathrm{m}$ donor-receiver substrate gap has been used with a reduced environmental pressure of 1 mbar. It is important to notice that for this application, a multilayer structure has been transferred in a single step without loss of device performance.

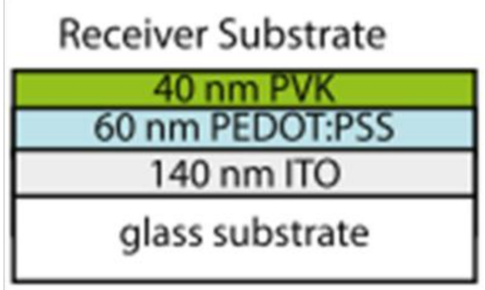

\begin{tabular}{|c|}
\hline Donor Substrate \\
\hline PFO (:dye) \\
\hline $80 \mathrm{~nm}$ metal \\
\hline $190 \mathrm{~nm}$ TP \\
\hline $\mathrm{SiO}_{2}$ substrate \\
\hline
\end{tabular}

Figure 6: Structures of the receiver and the donor substrates 
Different cathodes materials have been used to optimize the PLEDs performances: silver, aluminum and aluminum with an ultra-thin layer of the alkaline tetrabutylammonium hydroxide (TBA) directly deposited onto the electrode to favor electron injection ${ }^{40}$. Figure 7 shows microscope images of the LIFT printed RGB pixels with the Al and Al/TBA cathodes. We measured luminance as high as $540 \mathrm{~cd} . \mathrm{m}^{-2}$ for the blue pixel, $232 \mathrm{~cd} . \mathrm{m}^{-2}$ and $145 \mathrm{~cd} . \mathrm{m}^{-2}$ have also been obtained for the green and red pixels respectively. So, there is no doubt that laser printing is an appropriate process to realize PLED devices. Its ability to print multilayer films in a single step process without reduction of the material performances is a great advantage over other printing technologies.
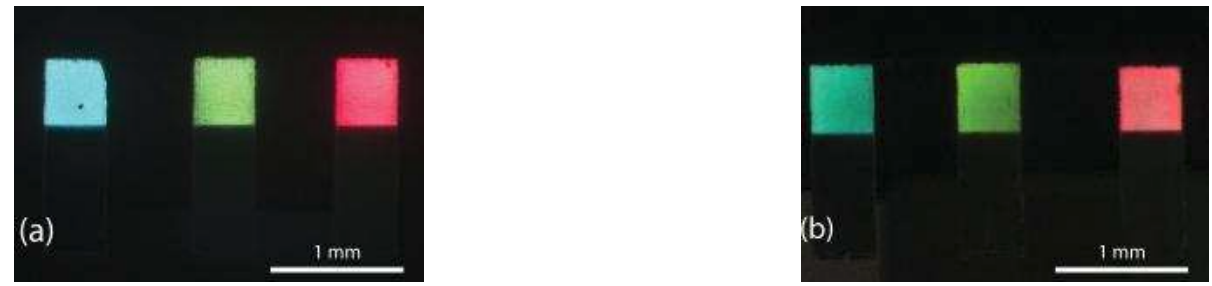

Figure 7: Tri-color pixels imaged using a light microscope are shown for an $\mathrm{Al} / \mathrm{TBA}$ cathode at $20 \mathrm{~V}$ (a) and for an $\mathrm{Al}$ cathode at $24 \mathrm{~V}(\mathrm{~b})$.

\section{APPLICATION TO ORGANIC THIN FILM TRANSISTORS}

The LIFT process has also been investigated for the realization of organic/inorganic thin film transistors (TFTs) ${ }^{14,41}$ and the results have been compared with those obtained with inkjet technology. In bottom gate configuration, the receiver substrate was silicon $(\mathrm{Si})$ covered by a $300 \mathrm{~nm}$ thick silicon dioxide $\left(\mathrm{SiO}_{2}\right)$ layer, and these materials play also the role of gate and dielectric of the transistors, respectively. Many different materials have been tested as organic semiconductors (PBTTT, PQT, P3HT, DS4T, diPhAc-3T, Pentacene, N2200) and for the fabrication of the source \& drain electrodes we used $\mathrm{Au}, \mathrm{Ag}$ or Pt which have been either evaporated or LITFed.

First, pixels of $80 \mathrm{~nm}$ thick PQT-12 (poly[5,5'-bis(3-alkyl-2-thienyl)-2,2'-bithiophene]) were printed on the receiver substrate, and the OTFTs were completed by thermally evaporated $50 \mathrm{~nm}$ thick gold lines through a mask as sourcedrain electrodes in a top contact (TC) configuration with specific width $(\mathrm{W}=400 \mu \mathrm{m})$ and channel lengths $(\mathrm{L}=30-300$ $\mu \mathrm{m})$. A mobility of $2.410^{-2} \mathrm{~cm}^{2} \cdot \mathrm{V}^{-1} \cdot \mathrm{s}^{-1}$ has been measured with a threshold voltage $\mathrm{V}_{\mathrm{T}}=-9 \mathrm{~V}$ and $\mathrm{I}_{\mathrm{On}} / \mathrm{I}_{\mathrm{Off}}$ ratio of $310^{3}$. The comparison of these results with the characteristics of inkjet-printed OTFT with similar materials and configuration shows that laser printing can lead to the fabrication of devices with a better or a worse mobility compared to inkjet, depending of the material. However, we also observed that the current ratio is always lower for laser printed OTFTs, around $10^{3}$ instead of $10^{5}$ for inkjet printing. This result shows that LIFT process induces some modifications to the organic semiconducting layer which are detrimental to the device electrical performances.

Then, to illustrate the interest of laser printing of solid, we have fabricated an OTFT based on a no-soluble organic semiconductor (OSC), and then this material cannot be used by other digital printing technologies. We used the p-type organic semiconductor, distyryl-quaterthiophene (DS4T) which was vacuum-deposited on a donor substrate and transferred on $\mathrm{Si} / \mathrm{SiO}_{2}$-based receiver substrate. The transfer of this material requires the use of triazene DRL. Those transistors exhibit a mobility of $0.02 \mathrm{~cm}^{2} . \mathrm{V}^{-1} \cdot \mathrm{s}^{-1}$ and a current ratio of $3.10^{3}$ which is still too low for industrial applications, but we demonstrated the high stability over time (100 days standard test) of the laser printed TFTs ${ }^{42}$.

At last, we used a new organic semiconductor the diPhAc-3 $\mathrm{T}^{43}$ (Bis(2-phenylethynyl end-substituted terthiophene). The Epitaxial thin-film growth of this material follows an island Volmer-Weber mechanism meaning that the films show distinctive 3D grains. The large intermolecular interaction involved in such growth mechanism makes the thin films less sensitive to the mechanical damages induced by the laser. Then, it was expected here that the high structural cohesion of diPhAc-3T-based grains will resist to laser transfer and to offer new mechanical properties to resulting printed pixels. Effectively, AFM analysis confirmed that the laser transfer didn't modify the material structure, and the printed pixel morphology was very good even when not using the triazene layer. The resulting OTFTs in TC configuration exhibited very good electrical performances $\left(\mu=0.04 \mathrm{~cm}^{2} / \mathrm{Vs}, \mathrm{V}_{\mathrm{T}}=0 \mathrm{~V}, \mathrm{I}_{\mathrm{On}} / \mathrm{I}_{\mathrm{Off}}=2.8 \times 10^{5}\right)$, and the stability tests over 100 days were also very good ${ }^{44}$.

Single step printing of multilayer film has also been investigated ${ }^{45}$ but this approach didn't provide any significant enhancement compared to the layer by layer technique, unlike that has been observed for PLED laser printing. Top gate TFTs have also been successfully printed to address the problem of deposition of dielectric layer ${ }^{46}$. 
To conclude, the realized bottom gate OTFTs by laser printing the organic semiconductors yielded high electrical performances. OTFTs with mobilities of a few $10^{-2} \mathrm{~cm}^{2} / \mathrm{Vs}$ and Ion/Ioff of the order of $10^{5}$ were realized. The performances of laser printed TFTs were comparable or even higher than those of the OTFTs realized by other techniques. However, laser printing process induces some mechanical stresses to the material and can modify its structural organization. LIFT process is then particularly attractive for printing organic materials with high structural cohesion.

\section{APPLICATION TO SENSORS}

To study the ability of laser printing process to be used for the fabrication of sensors and biosensors, we investigated four types of devices: two sensors and two biosensors which are described hereafter. One of the sensors as well as one of the biosensors is based on a special design relying on the properties of surface (SAW) and bulk acoustic waves (BAW). These systems will not be described here but a complete description has already been published ${ }^{47,48}$

\subsection{Chemical sensors for organic compounds based on chemical-selective polymeric materials}

The purpose of this task was to fabricate a sensor array for monitoring volatile organic compounds (VOCs) by combining the sensitivity of specially designed SAW/BAW devices with the selectivity of special polymers and their combination in one device. Five different polymers (PEI, PIB, PECH, PScMA-me, HPMC) have been used as sensitive materials and the donor substrates were prepared by MAPLE (Matrix-assisted pulsed laser deposition) technique ${ }^{49}$. Then, the sensitivity of each laser printed polymer has been measured with five analytes and water $\left(\mathrm{Tol}, \mathrm{H}_{2} \mathrm{O}, \mathrm{DCP}\right.$, DMMP, DCM, EtOAc). As an example, Response curves for PECH, PEI, and PIB sensors upon exposure to different concentration of DMMP are shown on figure 8 . We can observe the linear response of the sensors and sensitivity of few tenths of ppm have been measured

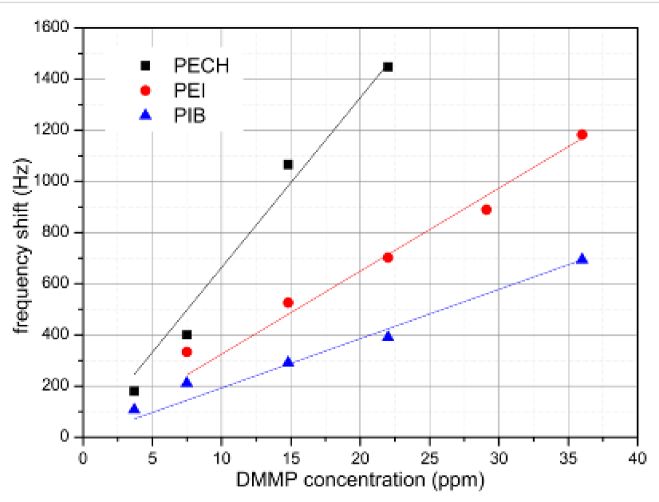

Figure 8: Response curves for PECH, PEI, and PIB sensors upon exposure to different concentration of DMMP

Then, we investigated the possibility of using the response of the different polymers to the same environment in order to increase the sensor selectivity. First, we printed three analytes (PIB, PEHC, PEI) to detect DMMP, DCM and EtOAc and characterized the sensor response. The Principal Component Analysis (PCA) has been performed from this study we can conclude that this sensor system can discriminate the three analytes, and that the discrimination increases at higher concentrations. Similar investigations have been done to determine the responses of 3 polymers (PIB, PECH, PEI) to 5 analytes and water (Tol, $\mathrm{H}_{2} \mathrm{O}$, DCP, DMMP, DCM, EA). We observed that this system can discriminate $\mathrm{H}_{2} \mathrm{O}$, DMMP, DCM and EA, but these three polymers cannot discriminate between Tol and DCP.

Then, we used five polymers (PIB, PECH, PEI, PScMA-me, HPMC) to discriminate the five analytes and water. The PCA analysis which let us to conclude that this sensor system based on five polymers can discriminate the water from Tol and DCP, and that the selectivity between Tol and DCP increases compared to the 3 polymer system.

\subsection{Gas sensor based on semiconducting oxide and/or catalyst}

The gas sensor structures used for the transfer evaluation are the standard gas sensor microstructures used by MICROSENS. These sensors comprise of a thin membrane with a heater element onto which the pixel was to be 
deposited. The sensitive material is a $\mathrm{SnO}_{2}$ semiconducting layer which is sensitive to oxidizing and/or reducing gases at optimized temperature. These gas sensors are currently used for air quality monitoring, detecting $\mathrm{CO}, \mathrm{NO}_{2}$, $\mathrm{VOC}$ (Volatile Organic Compounds) in air ${ }^{50}$.

Different approaches have been suggested and tested to prepare the donor substrates used to laser print the $\mathrm{SnO}_{2}$ film. The best results have been obtained with a precursor of $\mathrm{SnCl}_{2}(\mathrm{acac})_{2}{ }^{51}$. In the frame of the optimization of the transfer process $^{52}$, we also observed that the absence of the DRL does not influence the quality of the transfer. Since the $\mathrm{SnCl}_{2}(\mathrm{acac})_{2}$ based pixels show the most promise for the sensor fabrication, the evaluation of the sensor characteristics was performed using these types of pixels. Figure 9 shows a photograph of the LIFT-printed $\mathrm{SnO}_{2}$ pixel and the sensor response to different EtOH concentrations. Sensitivity lower than few ppm has been measured and the performances of the LIFT-printed sensors appear higher than those of the industrial gas sensors based on the same design and materials. This is probably due to a higher porosity of the printed material. Moreover, stability tests over time are also very promising, less than $25 \%$ losses in one year.

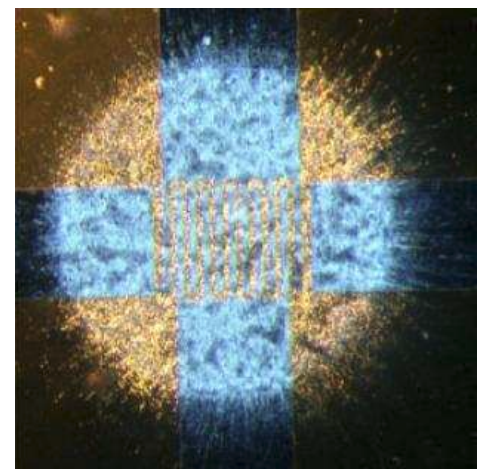

(a)

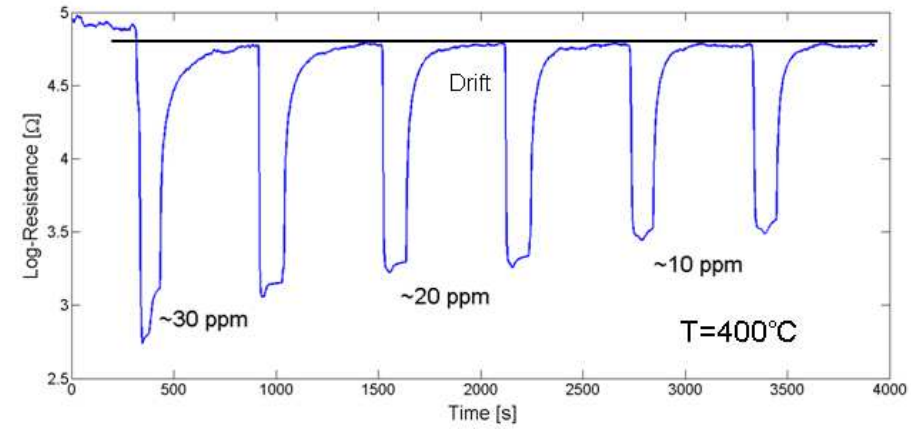

(b)

Figure 9: Transfers of $\mathrm{Sn}(\mathrm{acac})_{2} \mathrm{Cl}_{2}$ onto the Platinum inter-digitized electrodes (a) and signal of $\mathrm{SnO}_{2}$ from $\mathrm{Sn}(\mathrm{acac})_{2} \mathrm{Cl}_{2}$ to different EtOH concentrations (b)

\section{APPLICATION TO BIOSENSORS}

\subsection{SAW sensors based on proteins}

The purpose of this task was to realize biosensors based on a SAW device and combining the sensitivity of biomolecules. Three proteins: wild type bovine Odorant-Binding Protein (wtbOBO), double mutant bOBP (dmbOBP) and wild type pig OBP (wtpOBP), have been used to detect R-(-)-1-octen-3-ol (octenol) and R-(-)-carvone (carvone) molecules. The proteins have been transferred on sensor by means of LIFT process in liquid phase. As the surface sensor is composed of inter-digitized electrodes, a careful optimization was required to obtain a uniform coverage of the sensor by the proteins. The sensitivities obtained for octenol and carvone detection with LIFT-fabricated sensors are comparable to those obtained with other printing processes, demonstrating the feasibility of LIFT technique for fabrication of SAW biosensors based on OBPs. The frequency responses of the three sensors to different concentrations of octenol and carvone were analyzed by PCA, and this analysis reveals that the sensor system can discriminate the two odorants. A very good selectivity was obtained also at low concentrations.

\subsection{Electrochemical photosynthetic protein-based biosensors}

This part of the study was dedicated to the realization of biosensors by means of liquid phase LIFT of biomaterials on different sensing substrates. The specific target application is the high sensitivity detection of pesticides and herbicides in water and agro-food products. The principle of the biosensor is based on the electrochemical detection using photosynthetic proteins based on the properties of the thylakoid membrane. The proteins are printed on a screen printed gold electrodes (Au SPE). This electrode is placed in water and illuminated by the light from a pulsed LED. Under illumination the proteins generate electrons which are collected by the SPE and the intensity of the current represents the response of the sensor. When herbicides or pesticides are present in the water, they attach to the proteins and reduce the amount of electrons generated under illumination. Figure 10 shows a picture of the commercial apparatus from biosensor (10a), the scheme of principle of the system (10b) and a picture of the electrode with the proteins LIFT-printed onto one electrode (10c). 


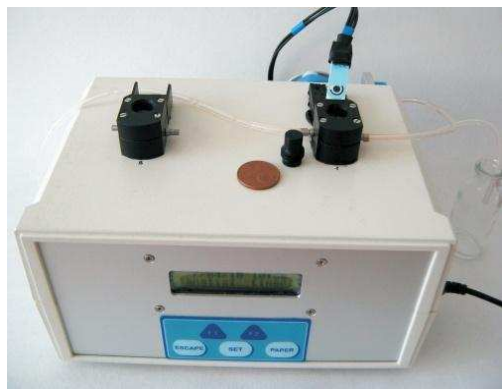

(a)

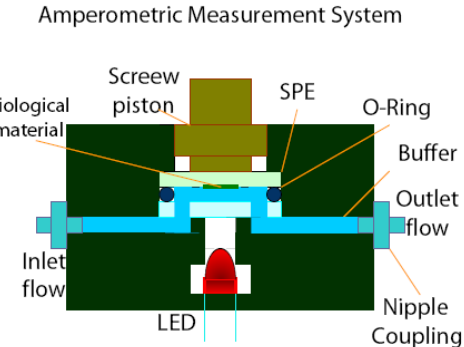

(b)

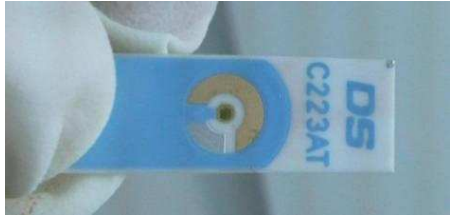

(c)

Figure 10: picture of the commercial apparatus from biosensor (a), the scheme of principle of the system (b) and a picture of the electrode with the proteins LIFT-printed onto one electrode (c)

The sensor characterization proves that only the laser printed thylakoids were successfully immobilized on the surface of the non-functionalised SPEs ${ }^{53}$. This is an important result which allows the elimination of the functionalisation step in biosensors preparation. In order to test the functionality of the laser printed photosynthetic biosensors and to optimize the process, we have tested common herbicides (Linuron, 3-(3,4-dichlorophenyl)-1-methoxy-1-methylurea) and diuron (3(3,4-dichlorophenyl)-1,1-dimethylurea). The limits of detection obtained for the Diuron and Linuron herbicides are $8 \mathrm{x}$ $10^{-9} \mathrm{M}$ and $4 \times 10^{-9} \mathrm{M}$, respectively. These values are better than those currently obtained with the standard printing techniques and the laser printing process does not require any functionnalization step. These very good results are due to the high impact pressure induced by the laser printing technique. In this process, the thylakoid proteins reach the gold SPE with a very high velocity which remove any air from the electrode porosity and ensure a high electrical connection between the biomaterial providing the electrons and the SPE collecting these charges ${ }^{54}$. The figure 11 illustrates this effect.

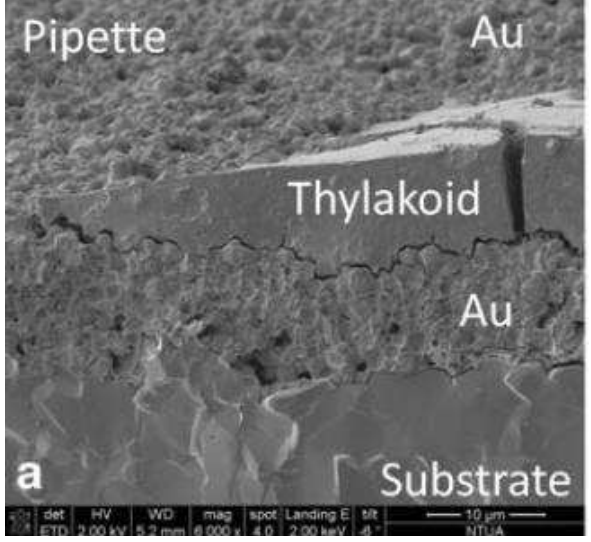

(a)

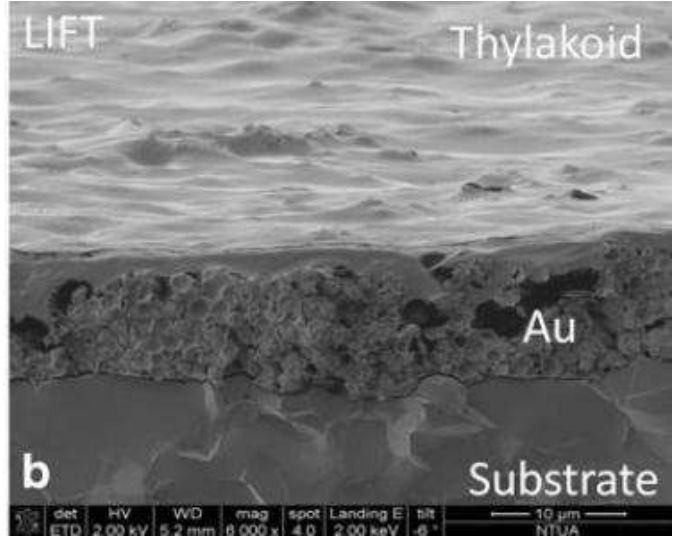

(b)

Figure 11: Cross section SEM images of gold electrodes covered by thylakoid membranes printed by a) the reference pipette method, b) the LIFT technique.

\section{APPLICATION TO ENERGY HARVESTING}

The last application we investigated with the LIFT process was dedicated to the fabrication of microgenerators for energy harvesting purpose, which is one of the key points for the realization of autonomous devices. Our study deals with the realization of thermoelectric (TE) microgenerators.

Suitable thermoelectric materials for the use at ambient temperatures are chalcogenides. Among these the most widely used materials are compounds of bismuth, antimony, selenium and tellurium. They provide high intrinsic Seebeck coefficients at room temperature. For the LIFT of thermoelectric materials we chose sputtering targets $\mathrm{Of} \mathrm{Bi}_{2} \mathrm{Se}_{3}$ and $\mathrm{Bi}_{2} \mathrm{Te}_{3}$ to produce donor films for LIFT. The two materials act as TE elements conducting as a p-type and n-type doped material. The donors have a thickness of $300 \mathrm{~nm}$ for BiSe and $750 \mathrm{~nm}$ for BiTe. 
The fabrication of an energy harvesting device required the printing of thick $(1 \mu \mathrm{m})$ thermoelectric film with an individual element surface area larger than several $\mathrm{mm}^{2}$. A paraffin/polyolefin blend (Parafilm) covering a glass substrate was used as a receiver, and we demonstrated the transfer of films with a maximal size of $\sim 15 \mathrm{~mm}^{2}$. The characterization of the LIFT-printed films was evaluated by comparing the donor and LIFTed pixels' Seebeck coefficients as a measure of the ability of thermoelectric conversion. The as-sputtered donor films of $\mathrm{Bi}_{2} \mathrm{Te}_{3}, \mathrm{Bi}_{2} \mathrm{Se}_{3}$ and $\mathrm{Bi}_{0.5} \mathrm{Sb}_{1.5} \mathrm{Te}_{3}$ had a Seebeck coefficient of $-64 \pm 1 \mu \mathrm{V} / \mathrm{K},-121 \pm 1 \mu \mathrm{V} / \mathrm{K}$ and $+177 \pm 8 \mu \mathrm{V} / \mathrm{K}$ respectively ${ }^{55}$. The LIFT process leads to a decrease in the Seebeck coefficient of these samples.

Nevertheless this damage is limited and the values for LIFTed pixels are good enough for the fabrication of thermoelectric elements. This decrease is attributed to the phase transformation of the irradiated side of the pixel. For the fabrication of a thermoelectric generator we first selected selected the lateral/lateral $\mathrm{W}$ design (figure 12b).

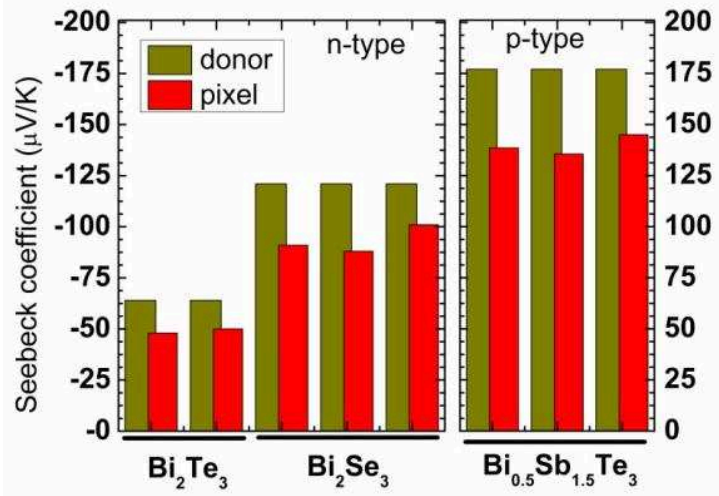

(a)

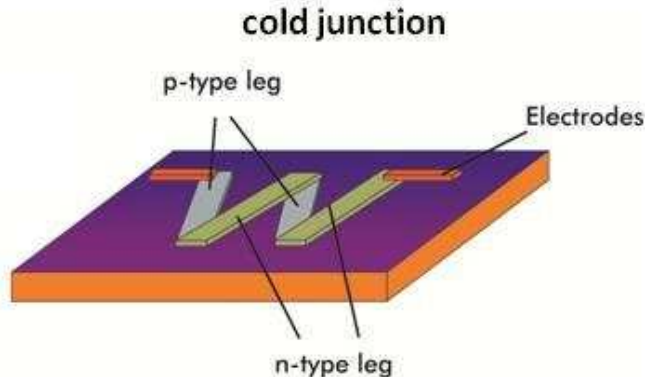

hot junction

(b)

Figure 12: Comparison of the Seebeck coefficients before (green-donor) and after (red-pixel) the LIFT process for the three materials used (a). TE generator designs that was used to validate the LIFT process (a)

The characterization of LIFT-printed thermoelectric microgenerators was performed was performed varying the number of legs. Seebeck coefficient was lower than expected from the previous Seebeck measurements of single LIFTed pixels. However the highest output voltage/thermocouple for a temperature gradient of $\sim 100 \mathrm{~K}$ was $\sim 7 \mathrm{mV}$. Hence, the number of 9-10 thermocouples of our devices provides an output voltage sufficiently high to drive commercially available electronic integrated circuits designated to operate in conjunction with such energy harvesting generators.

\section{CONCLUSION}

This work demonstrated the high potential of LIFT process to print a wide range of organic and inorganic materials in liquid and solid phase. The ability to transfer, in a single step a multilayer of thin film is of significant importance, and appears well adapted to the fabrication of PLED. We also demonstrated that this process can generate some mechanical stresses in the printed materials that could have deleterious effects on its electrical properties. However, some high performance OTFTs have been LIFT-printed. The most promising short term applications are clearly related to the manufacturing of sensors and biosensors. In both case, devices with higher performances than similar commercial sensors have been realized. This application does not required a high throughput system and a cost effective machine could be build to address this market and demonstrate the ability of LIFT process to be used as an industrial manufacturing tool. That would a first step towards a high throughput roll to roll LIFT machine ${ }^{56}$.

\section{ACKNOWLEDGMENTS}

Funding for the research performed in this e-LIFT project was provided by the European Commission $7^{\text {th }}$ Framework Programme (FP7-ICT project $\mathrm{N}^{\circ}$. 247868) 


\section{REFERENCES}

[1] Bohandy, J., Kim, B.F., and Adrian, F.J. "Metal deposition from a supported metal film using an excimer laser" J. Appl. Phys., 60 (4), 1538-1539 (1986)

[2] Arnold, C.B., Serra, P., and Pique, A. "Laser direct-write techniques for printing of complex materials" MRS Bull., 32, 23-31 (2007)

[3] Serra P., Colina M., Fernández-Pradas J. M., Sevilla L., Morenza J. L., "Preparation of functional DNA microarrays through laser-induced forward transfer" Appl. Phys. Lett. 85 (9) 1639 (2004)

[4] Koundourakis G., Rockstuhl C., Papazoglou D., Klini A., Zergioti I., Vainos N.A., Fotakis C., "Laser printing of active optical microstructures" Appl. Phys. Lett. 78 (7), 868 (2001)

[5] Kaur K.S., Subramanian A.Z., Ying Y.J., Banks D.P., Feinäugle M., Horak P., Apostopoulos V., Sones C.L., Mailis S., Eason R.W., "Waveguide mode filters fabricated using laser-induced forward transfer technique" Optics Express 19 (10), 9814-9819, (2011)

[6] Thomas B., Alloncle A.P., Delaporte Ph., Sentis M., Sanaur, Barret M., Collot Ph. "Experimental investigations of laser induced forward transfer process of organic thin films"Appl. Surf. Sci. 254 (4), 1206-1210 (2007)

[7] Zergioti I., Karaiskou A., Papazoglou D.G., Fotakis C., Kapsetaki M., Kafetzopoulos D., "Time resolved schlieren study of sub-picosecond and nanosecond laser transfer of biomaterials" Appl. Phys. Lett. 86163902 (2005)

[8] Doraiswamy A., Narayan R., Lippert T., Urech. L., Wokaun A., Nagel M., Hopp B., Dinescu M., Modi R., Auyeung R., Chrisey D., "Excimer laser forward transfer of mammalian cells using a novel triazene absorbing layer" Appl. Surf. Sci. 252 (13), 4743-4747 (2006)

[9] Boutopoulos C., Pandis C., Giannakopoulos K., Pissis P., Zergioti I., 'Polymer/carbon nanotube composite patterns via laser induced forward transfer' Appl. Phys. Lett. 96, 041104 (2010)

[10] Banks D.P., Grivas C., Mills J.D., Eason R.W., Zergioti I., "Nanodroplets deposited in microarrays by femtosecond Ti : sapphire laser-induced forward transfer" Appl. Phys. Lett. 89, 193107 (2006)

[11] Kim H., Auyeung R.C.Y., Lee S.H., Huston A.L., Piqué A., "Laser forward transfer of silver electrodes for organic thin-film transistors" Appl. Phys. A 96, 441-445 (2009)

[12] Rapp L., Ailuno J., Alloncle A.-P., Delaporte Ph., "Pulsed-laser printing of silver nanoparticles ink: control of morphological properties", Optics Express 19 (22), 21563-21574 (2011)

[13] Palla-Papavlu A., Dinca V., Paraico I., Moldovan A., Shaw-Stewart J., Schneider Ch., Kovacs E., Lippert Th., Dinescu M.. 'Microfabrication of polystyrene microbead arrays by laser induced forward transfer' J. of Appl. Phys. 108 (3), 033111 (2010)

[14]Rapp L., Diallo A.K., Alloncle A.P., Videlot-Ackerman C., Fages F., Delaporte Ph., 'Pulsed-laser printing of organic thin film transistors' Appl. Phys. Lett. 95, 171109 (2009)

[15]Fardel R., Nagel M., Nüesch F., Lippert T., Wokaun A., "Fabrication of organic light-emitting diode pixels by laser-assisted forward transfer" Appl. Phys. Lett. 91, 061103 (2007)

[16]Zergiotti I., Koudourakis G., Vainos N., Fotakis C., in Direct Write Technologies for Rapid Prototyping Applications (eds A. Pique and D.B. Chrisey), Academic Press, New York, 493-516 (2002)

[17] Kyrkis, K., Andreadaki, A., Papazoglou, D., Zergioti, I., in Recent Advances in Laser Processing of Materials (eds J. Perrière, E. Millon, and E. Fogarassy), Elsevier, 213-241. (2006)

[18] Sakat H., Wakaki M., [Nanomaterials; Processing and Characterization with Lasers], eds H. Zeng, C. Guo, W. Cai, S. C. Singh, Wiley-Blackwell, John Wiley \& Sons Publishers, Weinheim, 219-240 (2012)

[19] Wang Q., Sametoglu V., Yin Tsui Y., [Nanomaterials; Processing and Characterization with Lasers], eds H. Zeng, C. Guo, W. Cai, S. C. Singh, Wiley-Blackwell, John Wiley \& Sons Publishers, Weinheim, 241-254 (2012)

[20] Nagel M., Lippert T., [Nanomaterials; Processing and Characterization with Lasers], eds H. Zeng, C. Guo, W. Cai, S. C. Singh, Wiley-Blackwell, John Wiley \& Sons Publishers, Weinheim, 255-316 (2012)

[21] Shaw-Stewart J., Fardel R., Nagel M., Delaporte Ph., Rapp L., Cibert Ch., Alloncle A.P., Nuesch F., Lippert T., Wokaun A., 'The effect of different laser pulse lengths upon laser induced forward transfer using a triazene polymer as a sacrificial layer', J. of Optoelectronics and Advanced Materials (JOAM) 12 (3), 605 - 609, (2010) 
[22] Feinaeugle M., Alloncle A.P., Delaporte Ph., Sones C.L., Eason R.W., 'Time-resolved shadowgraph imaging of femtosecond laser-induced forward transfer of solid materials', Applied Surface Science 258, 8475-8483, (2012)

[23]Zergioti I., Papazoglou D.G., Karaiskou A., Fotakis C., Gamaly E., Rode A., 'A comparative schlieren imaging study between ns and sub-ps laser forward transfer of Cr', Applied Surface Science 208-209, 177-180 (2003)

[24] Fardel R., Nagel M., Nuesch F., Lippert T., Wokaun A., 'Shadowgraphy investigation of laser-induced forward transfer: Front side and back side ablation of the triazene polymer sacrificial layer', Applied Surface Science 255, 5430-5434, (2009)

[25] Rapp L., Cibert Ch., Alloncle A.P., Delaporte Ph., Nenon S., Videlot-Ackermann Ch., Fages F., “Comparative time resolved shadowgraphic imaging studies of nanosecond and picosecond laser transfer of organic materials.”, Proc. SPIE 7131L, (2009)

[26] Fardel R., Nagel M., Nuesch F., Lippert T., Wokaun, A. 'Laser-induced forward transfer of organic LED building blocks studied by time-resolved shadowgraphy', J. Phys. Chem. C 114, 5617-5636. (2010)

[27] Shaw-Stewart J., Chu B., Lippert T., Maniglio Y., Nagel M, Nüesch F., Wokaun A., 'Improved laser-induced forward transfer of organic semiconductor thin films by reducing the environmental pressure and controlling the substrate-substrate gap width', Appl Phys A 105, 713-722 (2011)

[28] Duocastella M., Fernández-Pradas J. M., Morenza J. L., Serra P., 'Time-resolved imaging of the laser forward transfer of liquids', J. Appl. Phys. 106, 084907 (2009)

[29] Duocastella M., Patrascioiu A., Fernández-Pradas J.M., Morenza J.L., Serra P., 'On the correlation between droplet volume and irradiation conditions in the laser forward transfer of liquids', Appl. Phys. A 109, 5-14 (2012)

[30] Brown M., Kattamis N., Arnold C. B., 'Time-resolved study of polyimide absorption layers for blister-actuated laser-induced forward transfer', J. of Appl. Phys. 107, 083103 (2010)

[31] Boutopoulos Ch., Alloncle A. P., Zergioti I., Delaporte Ph., 'A time-resolved shadowgraphic study of laser transfer of silver nanoparticle ink', Appl. Surf. Sci., doi:10.1016/j.apsusc.2012.12.002, (2012)

[32] T. Lippert, 'Laser application of polymers', Adv. Polym. Sci. 168, 51-246 (2004)

[33] T. Lippert, T. Dickinson, 'Chemical and spectroscopic aspects of polymer ablation: Special features and novel directions', Chem. Rev. 103, 453-485 (2003)

[34] M. Nagel, R. Hany, T. Lippert, M. Molberg, F.A. Nüesch, D. Rentsch, 'Aryltriazene photopolymers for UVlaser applications: Improved synthesis and photodecomposition study', Macromol. Chem. Phys. 208 (3), $277-$ 286 (2007)

[35] Nagel M., Fardel R., Feurer P.,·Häberli M., Nüesch F., Lippert T., Wokaun A., 'Aryltriazene photopolymer thin films as sacrificial release layers for laser-assisted forward transfer systems: study of photoablative decomposition and transfer behavior', Appl Phys A 92, 781-789 (2008)

[36] Sones C. L., Feinaeugle M., Sposito A., Gholipour B., Eason R. W., 'Laser-Induced Forward Transfer-printing of focused ion beam pre-machined crystalline magneto-optic yttrium iron garnet micro-discs', Optics Express 20 (14), 15171-15179 (2012)

[37] Kaur K.S., Feinäugle M., Banks D.P., Ou J.Y., Di Pietrantonio F., Verona E., Sones C.L., Eason R.W., 'Laserinduced forward transfer of focused ion beam pre-machined donors', Appl. Surf. Sci. 257 (15), 6650-6653 (2011)

[38] Shaw-Stewart J., Lippert Th., Nagel M., Nueesch F., Wokaun A., 'Laser-Induced Forward Transfer of Polymer Light-Emitting Diode Pixels with Increased Charge Injection', ACS Applied Materials \& Interfaces 3 (2), 309 316 (2011)

[39] Shaw-Stewart J., Lippert T., Nagel M., Nüesch F.' Wokaun A., 'Sequential printing by laser-induced forward transfer to fabricate a polymer light-emitting diode pixel', Appl. Mater. \& Interfaces 4, 3535-2541 (2012)

[40] Shaw-Stewart J., Lippert T., Nagel M., Nüesch F., Wokaun A., 'Red-green-blue polymer light-emitting diode pixels printed by optimized laser-induced forward transfer', Appl. Phys. Lett. 100, 203303 (2012)

[41]Zergioti I., Makrygianni M., Dimitrakis P., Normand P., Chatzandroulis S., 'Laser printing of polythiophene for organic electronics', Appl. Surf. Sci. 257 (12), 5148-5151 (2012)

[42] Rapp L., Diallo A. K., Nénon S., Alloncle A. P., Videlot-Ackermann Ch, Fages F., Nagel M., Lippert Th., Delaporte Ph., 'Laser printing of a semiconducting oligomer as active layer in organic thin film transistors: impact of a protecting triazene layer', Thin Solid Films 520, 3043-3047 (2012) 
[43] Diallo A.K., Videlot-Ackermann C., Marsal P., Brisset H., Fages F., Kumagai A., Yoshimoto N., Serein-Spirau F., Lere-Porte J.P., 'Acetylenic spacers in phenylene end-substituted oligothiophene core for highly air-stable organic field-effect transistors', Phys. Chem. Chem. Phys. 12, 3845-3851 (2010)

[44]Rapp L., Serein-Spirau F., Lere-Porte J-P., Alloncle A. P., Delaporte Ph., Fages F., Videlot-Ackermann C., 'Laser printing of air-stable high performing organic thin film transistors', Organic Electronics 13 (10), 2035$2041(2012)$

[45] Rapp L., Nénon S., Alloncle A-P., Videlot-Ackermann C., Fages F., Delaporte Ph., 'Multilayer Laser Printing for Organic Thin Film Transistors', Appl. Surf. Sci. 257, 5152-5155 (2011)

[46] Diallo A.K., Rapp L., Nenon S., Alloncle A.-P., Delaporte Ph., Fages F., Videlot-Ackermann Ch., 'Top gate copper phthalocyanine thin film transistors with laser-printed dielectric', Synthetic Metals 161, 888-893 (2011)

[47] Cannatà D., Benetti M., Di Pietrantonio F., Verona E., Palla-Papavlu A., Dinca V., Dinescu M., Lippert T., 'Nerve agent simulant detection by solidly mounted resonators (SMRs) polymer coated using laser induced forward transfer (LIFT) technique'. Sensors and Actuators B 173, 32-39, (2012)

[48]Di Pietrantonio F., Cannatà D., Benetti M., Verona E., Varriale A., Staiano M., D'Auria S., 'Detection of odorant molecules via surface acoustic wave biosensor array based on odorant-binding proteins.' Biosensors and Bioelectronics 41, 328-334, (2013)

[49] Palla-Papavlu A., Dinca V., Dinescu M., Di Pietrantonio F., Cannatà D., Benetti M., Verona E., 'Matrixassisted pulsed laser evaporation of chemoselective polymers', Appl Phys A 105, 651-659 (2011)

[50] Grisel A., Demarne V., 'Fabrication of Integrated Thin Film Semiconductor Gas Sensors', Chemical Sensor Technology 2, Kodansha Ltd T. Seiyama Edit Tokyo (1989)

[51] Mattle T., Hintennach A., Lippert T., Wokaun A., 'Laser induced forward transfer of $\mathrm{SnO}_{2}$ for sensing applications using different precursors systems', Appl. Phys. A 110 (2), 309-316 (2013)

[52] Mattle T., Shaw-Stewart J., Hintennach A., Schneider C., Lippert T., Wokaun A., 'Shadowgraphic investigations into the laser-induced forward transfer of different SnO2 precursor films', Appl. Surf. Sci. doi:10.1016/j.apsusc.2012.11.146 (2012)

[53] Boutopoulos C., Touloupakis E., Pezzotti I., Giardi M. T., Zergioti I., 'Direct laser immobilization of photosynthetic material on screen printed electrodes for amperometric biosensor', Appl. Phys. Lett, 98 (9), 093703 (2011)

[54] Touloupakis E., Boutopoulos C., Buonasera K., Zergioti I., Giardi M.T., 'A photosynthetic biosensor with enhanced electron transfer generation realized by laser printing technology', Analytical and Bioanalytical Chemistry, 402 (10), 3237-3244 (2012)

[55] Feinäugle M., Sones C.L., Koukharenko E., Gholipour B., Hewak D.W., Eason R.W., 'Laser-induced forward transfer of intact chalcogenide thin films: resultant morphology and thermoelectric properties', Applied Physics A DOI 10.1007/s00339-012-7491-4, (2012)

[56] Hennig G., Baldermann T, Nussbaum C., Rossier M., Brockelt A., Schuler L., Hochstein G., 'Lasersonic ® LIFT Process for Large Area Digital Printing', Journal of Laser Micro/Nanoengineering 7 (3), 289-305 (2012) 